\title{
Planning a cluster randomized trial with unequal cluster sizes: practical issues involving continuous outcomes Lydia Guittet $^{1,2}$, Philippe Ravaud ${ }^{1,2}$ and Bruno Giraudeau*3,4
}

Address: ${ }^{1}$ Département d'Epidémiologie, Biostatistique et Recherche Clinique, Groupe Hospitalier Bichat-Claude Bernard (AP-HP), Université Xavier Bichat, Paris, France, ${ }^{2}$ INSERM U 738, Université Paris 7, Paris, France, ${ }^{3}$ INSERM CIC 202; Université François Rabelais de Tours; CHRU de Tours, France and ${ }^{4}$ INSERM U 717, Université Paris 7, Paris, France

Email: Lydia Guittet - guittetl@free.fr; Philippe Ravaud - philippe.ravaud@bch.ap-hop-paris.fr; Bruno Giraudeau* - giraudeau@med.univtours.fr

* Corresponding author

Published: 12 April 2006

BMC Medical Research Methodology 2006, 6:17 doi:10.1186/147|-2288-6-17

This article is available from: http://www.biomedcentral.com/I47/-2288/6/17

(C) 2006 Guittet et al; licensee BioMed Central Ltd.

This is an Open Access article distributed under the terms of the Creative Commons Attribution License (http://creativecommons.org/licenses/by/2.0), which permits unrestricted use, distribution, and reproduction in any medium, provided the original work is properly cited.
Received: 25 October 2005

Accepted: 12 April 2006

\begin{abstract}
Background: Cluster randomization design is increasingly used for the evaluation of health-care, screeening or educational interventions. At the planning stage, sample size calculations usually consider an average cluster size without taking into account any potential imbalance in cluster size. However, there may exist high discrepancies in cluster sizes.
\end{abstract}

Methods: We performed simulations to study the impact of an imbalance in cluster size on power. We determined by simulations to which extent four methods proposed to adapt the sample size calculations to a pre-specified imbalance in cluster size could lead to adequately powered trials.

Results: We showed that an imbalance in cluster size can be of high influence on the power in the case of severe imbalance, particularly if the number of clusters is low and/or the intraclass correlation coefficient is high. In the case of a severe imbalance, our simulations confirmed that the minimum variance weights correction of the variation inflaction factor (VIF) used in the sample size calculations has the best properties.

Conclusion: Publication of cluster sizes is important to assess the real power of the trial which was conducted and to help designing future trials. We derived an adaptation of the VIF from the minimum variance weights correction to be used in case the imbalance can be a priori formulated such as "a proportion $(\gamma)$ of clusters actually recruit a proportion $(\tau)$ of subjects to be included $(\gamma$ $\leq \tau)^{\prime \prime}$.

\section{Background}

A cluster randomized trial involves randomizing social units or clusters of individuals rather than the individuals themselves. This design, which is increasingly being used for evaluating healthcare, screening and educational interventions presents specific constraints that must be considered during planning and analysis [1,2]. Indeed, the responses of individuals within a cluster tend to be more similar than those of individuals of different clusters, and we thus define the clustering effect as $1+(m-1) \rho$, where $m$ is the average number of subjects per cluster and $\rho$ the intraclass correlation coefficient (ICC). This clustering effect is used during the planning of cluster randomized trials as an inflation factor to increase the sample size required by an individual randomization trial. However, such an approach does not take into account variations in 
cluster size, which might differ greatly. Indeed, as illustrated by Kerry et al [3], cluster size may depend on, for example, (i) the potential of recruitment of the cluster (i.e., the number of subjects belonging to each cluster), (ii) the eligible fraction of subjects, which may vary among clusters, or (iii) the ability of physicians to recruit subjects within each cluster. Such an imbalance in cluster size reduces the power of the trial and has to be taken into account in the sample size calculation.

Kerry et al [3] assessed the theoretical efficacy of 3 weightings of the inflation factor but in the context of cluster level analysis, so summary statistics are estimated at the cluster level and the unit of analysis remains the cluster. Manatunga et al [4], however, assessed a correction on the basis of the assumed distribution of cluster sizes in the context of marginal models, but the authors' simulations covered a range of ICCs larger than those usually observed in cluster randomized trials.

Our aim was therefore to assess these proposed corrections in the framework of cluster randomized trials in which the unit of analysis remains the subject, embedded in the cluster. We first describe the random effects model used to simulate clustered data; then display the simulation design used to evaluate the loss of power due to imbalance in cluster size and the findings. Corrections of the variance inflation factor to allow for cluster size inequality evaluated by simulation and robustness of these corrections to misspecification of the ICC is assessed. practical guidelines for the planning stage of cluster randomized trials are drawn and perspectives for future research.

\section{Methods and results \\ Theoretical background}

The mixed effects model

Let us supposed a continuous outcome distributed according to the following mixed-effects model:

$Y_{i j k}=\theta_{i}+\beta_{i j}+\varepsilon_{i j k}$

where $Y_{i j k}$ is the observed response for the $k$ th subject in the $j$ th cluster of the $i$ th group, $\theta_{i}$ is the overall mean in the $i$ th group, $\beta_{i j}$ is the random effect associated with the cluster effect and $\varepsilon_{i j k}$ is the residual effect. The $\beta_{i j}$ and $\varepsilon_{i j k}$ are assumed to be independent and normally distributed as $\left(0 ; \sigma_{b}^{2}\right)$ and $\left(0 ; \sigma_{w}^{2}\right)$ respectively.

The ICC quantifies the degree of similarity between the responses of subjects in the same cluster and is defined as the proportion of the total outcome variation between clusters: $\rho=\frac{\sigma_{b}^{2}}{\sigma_{b}^{2}+\sigma_{w}^{2}}$

\section{Sample size calculations}

Considering $g$ clusters of $m$ individuals to be randomized in each group, the total number of subject $N$ per group is given by [2]:

$N=m g=\frac{2 \sigma^{2}\left(t_{(1-\alpha / 2), 2(g-1)}+t_{(1-\beta), 2(g-1)}\right)^{2}[1+(m-1) \rho]}{\Delta^{2}}$

where $\Delta$ is the absolute mean difference between groups (i.e., $\left.\Delta=\left|\theta_{0}-\theta_{1}\right|\right), \sigma^{2}$ is the total variance defined as $\left(\sigma_{b}^{2}\right.$ $\left.+\sigma_{w}^{2}\right)$ and $t_{(1-\alpha / 2), 2(g-1)}$ and $t_{(1-\beta), 2(g-1)}$ is the $100 \times(1-$ $\alpha / 2)$ and $100 \times(1-\beta)$ percentiles of the Student $t$-distribution with $2(g-1)$ degrees of freedom. Considering the effect size, defined as the relative difference between groups (i.e., $E S=\left|\theta_{0}-\theta_{1}\right| / \sigma=\Delta / \sigma$ ), expression (3) can be re-written as:

$N=\frac{2\left(t_{(1-\alpha / 2), 2(g-1)}+t_{(1-\beta), 2(g-1)}\right)^{2}[1+(m-1) \rho]}{E S^{2}}$

\section{Impact of cluster size inequality}

\section{Simulation study}

Monte Carlo simulations were used to assess the impact of imbalance in cluster size on both power and type I error. A $2 \times 4 \times 4$ factorial plan was used, considering 2 effect sizes $(0.25,0.50)$ to be detected with fixed numbers of clusters $(5,10,20,40)$ and 4 a priori postulated values of the ICC $(0.005,0.02,0.05,0.10)$. The ICC values were chosen according to previously published estimates [5$15]$, and the number of clusters is in agreement with that from a recent review of cluster randomized trials in primary care settings in which the median number of randomized clusters was estimated at 34 [13]. The $\alpha$ and $\beta$ values were fixed at 0.05 and 0.20 , respectively, in any case.

Once the sample size was calculated, correlated data were simulated, according to model (1). From a practical point of view, data were generated as the sum of a fixed effect $\left(\theta_{0}\right.$ or $\theta_{1}$ if the control or experimental group, respectively) and realizations of the 2 random variables $\beta_{i j}$ and $\varepsilon_{i j k}$. For convenience and without loss of generality we set $\theta_{0}$ equal to 0 and $\left(\sigma_{b}^{2}+\sigma_{w}^{2}\right)$ equal to 1 . These constraints then allow for defining $\theta_{1}$ as the effect size ES, $\sigma_{b}^{2}$ as $\rho$ and $\sigma_{w}^{2}$ as $(1-\rho)$. 


\section{Cluster size}

For any combination of $E S, g$ and $\rho$, we simulated randomized trials with, on the one hand, constant cluster size and, on the other, imbalance in cluster size. In the absence of cluster sizes publications, three types of imbalance were considered:

\section{A moderate imbalance:}

For each group, each of the $N$ subjects had an equiprobability of being in any of the $g$ clusters randomized in this group. From a practical point of view, for any of the $N$ subjects, we randomly selected with equiprobability the cluster to which it belongs, before adding the appropriate realizations of random variables $\beta_{i j}$ and $\varepsilon_{i j k}$.

\section{A "Pareto" imbalance}

Following the economic Pareto's principle, we considered the situation in which $80 \%$ of the subjects actually belong to only $20 \%$ of the clusters. From a practical point of view, we thus defined 2 strata within each group: the strata of large clusters (e.g., 20\% of the $g$ clusters) and the strata of small clusters. Eighty percent of the $N$ subjects were in the large cluster strata, while the 20\% remaining were in the small cluster strata. Then, within each stratum, subjects were randomly assigned with equiprobability to one of the clusters.

\section{A Poisson imbalance}

Cluster sizes were finally defined according to a Poisson distribution, which has already been used in such a context $[16,17]$. We thus considered a Poisson distribution with parameter $m$ defined as $N / g$ and defined the cluster size of any cluster before generating the associated observations.

In this latter situation, and contrary to the 2 previous ones, the total number of patients per group varies and is equal to $N$ only on average. Moreover, in the 3 types of cluster size inequality, the actual number of clusters per group could be smaller than $g$, because clusters could be empty.

For any combination of ES, $g$ and ICC, and for any situation (balance or any type of imbalance in cluster size), 5000 replications of data were simulated by use of SAS 8.1 software.

\section{Analysis}

Data analysis involved no stratification on cluster size. We used the MIXED procedure in SAS [18,19] to assess restricted maximum likelihood (REML) estimates of variance components. The Wald test statistic was then used to test the significance of the intervention effect with the Student $t$-distribution, with $\mathrm{g}_{0}+\mathrm{g}_{1}-2$ degrees of freedom as the reference distribution, where $\mathrm{g}_{0}$ and $\mathrm{g}_{1}$ are the actual numbers of nonempty clusters in the control and intervention groups, respectively.

The empirical type I error and power were calculated as the proportion of significant trials (defined as a $\mathrm{p}$ value smaller than the nominal $\alpha$ level) when $\theta_{1}$ equals 0 and $E S$, respectively.

\section{Results}

Results are expressed as absolute bias and mean square error on the one hand, and empirical' type I error and power on the other. Table 1 displays the results associated with an a priori postulated effect size of 0.25 , while Table 2 displays the results associated with a 0.50 effect size. In 7 situations, data sets could not be generated for the following combinations ES/ICC/g: 0.25/0.020/5, 0.25/ $0.050 / 5,0.25 / 0.050 / 10,0.25 / 0.100 / 5,0.25 / 0.100 / 10$, $0.25 / 0.100 / 20$ and $0.50 / 0.100 / 5$. Indeed, when the number of clusters is small and/or the ICC high, even an infinite cluster size may not allow for achieving $80 \%$ power [20].

No significant bias was induced by inequality in cluster size (since the relative bias was no more than about $1.5 \%$, in absolute value), while the mean square error was barely increased in cases of severe imbalance (Pareto imbalance).

When the number of clusters is small, type I errors were estimated at a lower level than the nominal one, even with no imbalance in cluster sizes. A symmetrical result was also observed for power, which was estimated at a lower level than the nominal one. This result was of greater magnitude for small ICCs and for greater effect size, which corresponded to situations in which the total number of subjects to be included is reduced. Otherwise, although moderate and Poisson imbalances were of no influence, a Pareto's imbalance was associated with an increase in both type I and type II errors. As an example, if one is willing to detect a 0.25 effect size and plan a randomized trial with 10 clusters per arm with an a priori postulated ICC of 0.02, a Pareto imbalance leads to type I and type II errors of $9 \%$ and $38 \%$, respectively, and nominal values fixed at $5 \%$ and $20 \%$. This result is of greater magnitude for large ICCs and a small number of clusters.

Thus, while moderate imbalances (based on an equiprobability hypothesis) and Poisson's imbalances can be neglected at the planning stage, a more severe imbalance (such as the Pareto's imbalance) should be taken into account, thus leading to an adjustment in sample size calculations. 
Table I: Bias, mean square error, empirical type I error and power in cluster randomized trials according to several types of imbalance in cluster size - Effect size $=\mathbf{0 . 2 5}$

\begin{tabular}{|c|c|c|c|c|c|c|c|}
\hline \multicolumn{3}{|c|}{ Simulation parameters $\mid$} & \multirow{2}{*}{$\begin{array}{l}\text { Type of } \\
\text { imbalance }\end{array}$} & \multirow[t]{2}{*}{ Bias } & \multirow{2}{*}{$\begin{array}{c}\text { Mean Square } \\
\text { Error }\end{array}$} & \multirow{2}{*}{$\begin{array}{c}\text { Empirical } \\
\text { type I error }{ }^{2}\end{array}$} & \multirow{2}{*}{$\begin{array}{c}\text { Empirical } \\
\text { power }^{2}\end{array}$} \\
\hline $\begin{array}{c}\text { Intraclass } \\
\text { correlation } \\
\text { coefficient }(\rho)\end{array}$ & $\begin{array}{l}\text { Number of } \\
\text { clusters in } \\
\text { each arm (g) }\end{array}$ & $\begin{array}{l}\text { Total number } \\
\text { of subjects in } \\
\text { each arm }(N)\end{array}$ & & & & & \\
\hline \multirow[t]{4}{*}{0.005} & 5 & 485 & None & -0.0020 & 0.0062 & 0.0328 & 0.7756 \\
\hline & & & Moderate & -0.0015 & 0.0062 & 0.0300 & 0.7800 \\
\hline & & & Poisson & 0.0003 & 0.0061 & 0.0368 & 0.7814 \\
\hline & & & Pareto & 0.0002 & 0.0100 & 0.0664 & 0.6432 \\
\hline \multirow[t]{4}{*}{0.005} & 10 & 326 & None & 0.0005 & 0.0070 & 0.0326 & 0.7868 \\
\hline & & & Moderate & 0.0009 & 0.0073 & 0.0402 & 0.7838 \\
\hline & & & Poisson & -0.0010 & 0.0070 & 0.0356 & 0.7884 \\
\hline & & & Pareto & 0.0043 & 0.0100 & 0.0566 & 0.6968 \\
\hline \multirow[t]{4}{*}{0.005} & 20 & 282 & None & -0.0010 & 0.0072 & 0.0320 & 0.7942 \\
\hline & & & Moderate & 0.0014 & 0.0075 & 0.0398 & 0.7878 \\
\hline & & & Poisson & -0.0010 & 0.0076 & 0.0408 & 0.7802 \\
\hline & & & Pareto & 0.0000 & 0.0090 & 0.0486 & 0.7258 \\
\hline \multirow[t]{4}{*}{0.005} & 40 & 265 & None & 0.0006 & 0.0078 & 0.0444 & 0.7848 \\
\hline & & & Moderate & 0.0011 & 0.0082 & 0.0458 & 0.7936 \\
\hline & & & Poisson & -0.0017 & 0.0082 & 0.0484 & 0.7772 \\
\hline & & & Pareto & 0.0000 & 0.0086 & 0.0466 & 0.7572 \\
\hline \multirow[t]{4}{*}{0.020} & 10 & 629 & None & -0.0017 & 0.0070 & 0.0448 & 0.8012 \\
\hline & & & Moderate & 0.0017 & 0.0073 & 0.0544 & 0.7974 \\
\hline & & & Poisson & 0.0009 & 0.0074 & 0.0510 & 0.7992 \\
\hline & & & Pareto & -0.0022 & 0.0118 & 0.0904 & 0.6236 \\
\hline \multirow[t]{4}{*}{0.020} & 20 & 353 & None & -0.0004 & 0.0073 & 0.0452 & 0.8000 \\
\hline & & & Moderate & 0.0006 & 0.0074 & 0.0408 & 0.7980 \\
\hline & & & Poisson & -0.0007 & 0.0075 & 0.0458 & 0.7968 \\
\hline & & & Pareto & 0.0009 & 0.0115 & 0.0660 & 0.6546 \\
\hline \multirow[t]{4}{*}{0.020} & 40 & 290 & None & 0.0017 & 0.0080 & 0.0518 & 0.7932 \\
\hline & & & Moderate & 0.0001 & 0.0077 & 0.0466 & 0.7944 \\
\hline & & & Poisson & -0.0003 & 0.0077 & 0.0466 & 0.7912 \\
\hline & & & Pareto & 0.0003 & 0.0101 & 0.0556 & 0.7008 \\
\hline \multirow[t]{4}{*}{0.050} & 20 & 743 & None & -0.0007 & 0.0075 & 0.0436 & 0.7916 \\
\hline & & & Moderate & -0.0018 & 0.0077 & 0.0540 & 0.8026 \\
\hline & & & Poisson & -0.0003 & 0.0078 & 0.0536 & 0.7950 \\
\hline & & & Pareto & -0.0022 & 0.0115 & 0.0562 & 0.6256 \\
\hline \multirow[t]{4}{*}{0.050} & 40 & 361 & None & -0.0012 & 0.0080 & 0.0528 & 0.7944 \\
\hline & & & Moderate & 0.0031 & 0.0080 & 0.0510 & 0.7926 \\
\hline & & & Poisson & -0.0001 & 0.0080 & 0.0502 & 0.7904 \\
\hline & & & Pareto & -0.0023 & 0.0121 & 0.0604 & 0.6242 \\
\hline \multirow[t]{4}{*}{0.100} & 40 & 652 & None & 0.0021 & 0.0076 & 0.0504 & 0.7966 \\
\hline & & & Moderate & 0.0013 & 0.0078 & 0.0458 & 0.8118 \\
\hline & & & Poisson & -0.0022 & 0.0078 & 0.0506 & 0.7946 \\
\hline & & & Pareto & -0.0031 & 0.0121 & 0.0546 & 0.6006 \\
\hline
\end{tabular}

IN is the number of subjects per intervention arm, calculated under the assumption of constant cluster size

2 The nominal values for type I and type II error rates were fixed at 0.05 and 0.20 , respectively. 
Table 2: Bias, mean square error, empirical type I error and power in cluster randomized trials according to several types of imbalance in cluster size - Effect size $=\mathbf{0 . 5 0}$

\begin{tabular}{|c|c|c|c|c|c|c|c|}
\hline \multicolumn{3}{|c|}{ Simulation parameters $\mid$} & \multirow{2}{*}{$\begin{array}{l}\text { Type of } \\
\text { imbalance }\end{array}$} & \multirow[t]{2}{*}{ Bias } & \multirow{2}{*}{$\begin{array}{l}\text { Mean Square } \\
\text { Error }\end{array}$} & \multirow{2}{*}{$\begin{array}{l}\text { Empirical } \\
\text { type I error }\end{array}$} & \multirow{2}{*}{$\begin{array}{c}\text { Empirical } \\
\text { power }^{2}\end{array}$} \\
\hline $\begin{array}{l}\text { Intraclass cor- } \\
\text { relation coef- } \\
\text { ficient }(\rho)\end{array}$ & $\begin{array}{l}\text { Number of } \\
\text { clusters in } \\
\text { each arm (g) }\end{array}$ & $\begin{array}{l}\text { Total number } \\
\text { of subjects in } \\
\text { each arm }(N)\end{array}$ & & & & & \\
\hline \multirow[t]{4}{*}{0.005} & 5 & 89 & None & 0.0025 & 0.0238 & 0.0190 & 0.7648 \\
\hline & & & Moderate & 0.0010 & 0.0243 & 0.0204 & 0.7622 \\
\hline & & & Poisson & 0.0014 & 0.0243 & 0.0214 & 0.7596 \\
\hline & & & Pareto & -0.0064 & 0.0393 & 0.0256 & 0.6250 \\
\hline \multirow[t]{4}{*}{0.005} & 10 & 73 & None & -0.0011 & 0.0288 & 0.0328 & 0.7660 \\
\hline & & & Moderate & -0.0015 & 0.0290 & 0.0322 & 0.7718 \\
\hline & & & Poisson & -0.0007 & 0.0298 & 0.0318 & 0.7662 \\
\hline & & & Pareto & -0.0005 & 0.0344 & 0.0352 & 0.7090 \\
\hline \multirow[t]{4}{*}{0.005} & 20 & 67 & None & -0.0011 & 0.0303 & 0.0384 & 0.7764 \\
\hline & & & Moderate & -0.0038 & 0.0296 & 0.0318 & 0.7700 \\
\hline & & & Poisson & -0.0012 & 0.0301 & 0.0382 & 0.7664 \\
\hline & & & Pareto & -0.0004 & 0.0323 & 0.0334 & 0.7322 \\
\hline \multirow[t]{4}{*}{0.005} & 40 & 65 & None & 0.0005 & 0.0310 & 0.0446 & 0.7986 \\
\hline & & & Moderate & 0.0021 & 0.0322 & 0.0478 & 0.7896 \\
\hline & & & Poisson & 0.0028 & 0.0305 & 0.0396 & 0.7860 \\
\hline & & & Pareto & -0.0007 & 0.0320 & 0.0382 & 0.7518 \\
\hline \multirow[t]{4}{*}{0.020} & 5 & 119 & None & 0.0025 & 0.0238 & 0.0190 & 0.7648 \\
\hline & & & Moderate & -0.0011 & 0.0248 & 0.0310 & 0.7856 \\
\hline & & & Poisson & -0.0021 & 0.0250 & 0.0306 & 0.7786 \\
\hline & & & Pareto & 0.0009 & 0.0413 & 0.0674 & 0.6262 \\
\hline \multirow[t]{4}{*}{0.020} & 10 & 81 & None & -0.0031 & 0.0273 & 0.0320 & 0.7798 \\
\hline & & & Moderate & 0.0006 & 0.0282 & 0.0364 & 0.7772 \\
\hline & & & Poisson & -0.0003 & 0.0288 & 0.0378 & 0.7778 \\
\hline & & & Pareto & 0.0078 & 0.0394 & 0.0550 & 0.6910 \\
\hline \multirow[t]{4}{*}{0.020} & 20 & 70 & None & 0.0026 & 0.0312 & 0.0476 & 0.7838 \\
\hline & & & Moderate & 0.0026 & 0.0312 & 0.0476 & 0.7838 \\
\hline & & & Poisson & 0.0032 & 0.0306 & 0.0436 & 0.7894 \\
\hline & & & Pareto & 0.0003 & 0.0362 & 0.0460 & 0.7056 \\
\hline \multirow[t]{4}{*}{0.020} & 40 & 66 & None & -0.0026 & 0.0314 & 0.0498 & 0.7878 \\
\hline & & & Moderate & 0.0026 & 0.0312 & 0.0476 & 0.7838 \\
\hline & & & Poisson & 0.0049 & 0.0326 & 0.0476 & 0.7828 \\
\hline & & & Pareto & -0.0007 & 0.0337 & 0.0422 & 0.7328 \\
\hline \multirow[t]{4}{*}{0.050} & 5 & 423 & None & 0.0015 & 0.0246 & 0.0482 & 0.7980 \\
\hline & & & Moderate & -0.0001 & 0.0240 & 0.0460 & 0.8004 \\
\hline & & & Poisson & -0.0005 & 0.0237 & 0.0478 & 0.7988 \\
\hline & & & Pareto & 0.0026 & 0.0337 & 0.0768 & 0.6808 \\
\hline \multirow[t]{4}{*}{0.050} & 10 & 103 & None & -0.0006 & 0.0280 & 0.0426 & 0.7964 \\
\hline & & & Moderate & 0.0012 & 0.0286 & 0.0446 & 0.7952 \\
\hline & & & Poisson & -0.0017 & 0.0293 & 0.0440 & 0.7754 \\
\hline & & & Pareto & -0.0022 & 0.0466 & 0.0770 & 0.6342 \\
\hline \multirow[t]{4}{*}{0.050} & 20 & 76 & None & 0.0027 & 0.0298 & 0.0436 & 0.8020 \\
\hline & & & Moderate & -0.0018 & 0.0308 & 0.0452 & 0.7784 \\
\hline & & & Poisson & -0.0016 & 0.0323 & 0.0526 & 0.7672 \\
\hline & & & Pareto & -0.0056 & 0.0396 & 0.0528 & 0.6620 \\
\hline
\end{tabular}


Table 2: Bias, mean square error, empirical type I error and power in cluster randomized trials according to several types of imbalance in cluster size - Effect size $=\mathbf{0 . 5 0}$ (Continued)

\begin{tabular}{|c|c|c|c|c|c|c|c|}
\hline \multirow[t]{4}{*}{0.050} & 40 & 67 & None & -0.0019 & 0.0313 & 0.0468 & 0.7880 \\
\hline & & & Moderate & -0.0012 & 0.0294 & 0.0516 & 0.7740 \\
\hline & & & Poisson & 0.0000 & 0.0335 & 0.0504 & 0.7712 \\
\hline & & & Pareto & 0.0022 & 0.0376 & 0.0516 & 0.7026 \\
\hline \multirow[t]{4}{*}{0.100} & 10 & 213 & None & 0.0006 & 0.0263 & 0.0426 & 0.8056 \\
\hline & & & Moderate & 0.0015 & 0.0289 & 0.0530 & 0.7940 \\
\hline & & & Poisson & -0.0007 & 0.0287 & 0.0538 & 0.8004 \\
\hline & & & Pareto & -0.0027 & 0.0438 & 0.0730 & 0.6394 \\
\hline \multirow[t]{4}{*}{0.100} & 20 & 89 & None & -0.0029 & 0.0303 & 0.0470 & 0.7888 \\
\hline & & & Moderate & -0.0020 & 0.0324 & 0.0530 & 0.7760 \\
\hline & & & Poisson & -0.0004 & 0.0316 & 0.0488 & 0.7744 \\
\hline & & & Pareto & 0.0064 & 0.0492 & 0.0674 & 0.6276 \\
\hline \multirow[t]{4}{*}{0.100} & 40 & 70 & None & 0.0038 & 0.0331 & 0.0510 & 0.7890 \\
\hline & & & Moderate & 0.0031 & 0.0337 & 0.0506 & 0.7738 \\
\hline & & & Poisson & 0.0020 & 0.0332 & 0.0456 & 0.7658 \\
\hline & & & Pareto & 0.0019 & 0.0433 & 0.0536 & 0.6641 \\
\hline
\end{tabular}

IN is the number of subjects per intervention arm, calculated under the assumption of constant cluster size

2 The nominal values for type I and type II error rates were fixed at 0.05 and 0.20 , respectively.

\section{Sample size adjustment for unbalanced trials}

\section{Adjusted variance inflation factors}

The $(1+(m-1) \rho)$ factor in expressions (3) and (4) defines the variance inflation factor (VIF) that takes into account the correlation induced by the cluster randomization. This VIF supposes a constant cluster size $(m)$ or is based on the average cluster size in case of imbalance. Kerry et al [3] and Manatunga et al [4] proposed to adjust the VIF in cases of an imbalance in cluster size. Thus, we propose 4 corrections. The first 3 are based on weights derived from the $a$ priori postulated distribution of cluster sizes among the $\mathrm{g}$ clusters (i.e., the different values of $m_{j}$, where $m_{j}$ is the size of the $j^{\text {th }}$ cluster), and the fourth is based on the expected mean and variance of this latter distribution.

1. Equal weights (denoted $\left.w_{1}\right)[3]$ :

$V I F_{w_{1}}=\frac{\bar{m}}{g} \sum_{j=1}^{g} \frac{1}{m_{j}}(1-\rho)+\bar{m} \rho$ where $\bar{m}=\frac{1}{g} \sum_{j=1}^{g} m_{j}$

2. Cluster size weights (denoted $\left.w_{2}\right)[3]$ :

$V I F_{w_{2}}=1+\left(\overline{m_{A}}-1\right) \rho$ where $\overline{m_{A}}=\frac{\sum_{j=1}^{g} m_{j}^{2}}{\sum_{j=1}^{g} m_{j}}$

3. Minimum variance weights (denoted $w_{3}$ ) [3]:

$$
V I F_{w_{3}}=\frac{\bar{m} g}{\sum_{j=1}^{g} \frac{m_{j}}{1+\left(m_{j}-1\right) \rho}}
$$

4. Distribution-based correction (denoted d) [4]:

$V I F_{d}=1+\left(\frac{E(m)^{2}+\operatorname{var}(m)}{E(m)}-1\right) \rho$

where $\mathrm{E}(m)$ and $\operatorname{var}(m)$ are the expected mean and the variance of the cluster size.

We considered these 4 adjustments when a Pareto's imbalance is a priori supposed to be observed. Since moderate imbalances have been shown to be of no influence, we assumed a constant cluster size within each stratum associated with the Pareto's imbalance. The adjusted VIF then becomes (Appendix A):

$V I F_{w_{1}}=3.25+\left(\bar{m}_{w_{1}}-3.25\right) \rho$

with $\bar{m}_{w_{1}}=\frac{6.5(1-\rho) T^{2}}{g E S^{2}-2 \rho T^{2}}$ and $T=t_{(1-\alpha / 2), 2(g-1)}+t_{(1-\beta), 2(g}$

- 1)

$V I F_{w_{2}}=1+\left(3.25 \bar{m}_{w_{2}}-1\right) \rho$ 
Table 3: Required sample size and empirical Type I error and power when using corrected variance inflation factors with an a priori hypothesized Pareto imbalance in cluster size - Effect size $=0.25$

\begin{tabular}{|c|c|c|c|c|c|c|c|c|c|c|c|c|c|}
\hline \multirow{3}{*}{$\begin{array}{c}\text { Intraclass } \\
\text { correlation } \\
\text { coefficient }(\rho)\end{array}$} & \multirow{3}{*}{$\begin{array}{l}\text { Number of } \\
\text { clusters in } \\
\text { each arm (g) }\end{array}$} & \multicolumn{3}{|c|}{ No correction } & \multicolumn{3}{|c|}{ Equal weights } & \multicolumn{3}{|c|}{ Cluster size weights! } & \multicolumn{3}{|c|}{ Minimum variance weights } \\
\hline & & \multirow[t]{2}{*}{$\begin{array}{l}\text { Sample } \\
\text { size }\end{array}$} & \multicolumn{2}{|c|}{$\begin{array}{c}\text { Empirical } \\
\text { probabilities }\end{array}$} & \multirow[t]{2}{*}{$\begin{array}{c}\text { Sample } \\
\text { size }\end{array}$} & \multicolumn{2}{|c|}{$\begin{array}{c}\text { Empirical } \\
\text { probabilities }\end{array}$} & \multirow[t]{2}{*}{$\begin{array}{l}\text { Sample } \\
\text { size }\end{array}$} & \multicolumn{2}{|c|}{$\begin{array}{c}\text { Empirical } \\
\text { probabilities }\end{array}$} & \multirow[t]{2}{*}{$\begin{array}{l}\text { Sample } \\
\text { size }\end{array}$} & \multicolumn{2}{|c|}{$\begin{array}{c}\text { Empirical } \\
\text { probabilities }\end{array}$} \\
\hline & & & $\begin{array}{l}\text { Type I } \\
\text { error }\end{array}$ & Power & & $\begin{array}{l}\text { Type I } \\
\text { error }\end{array}$ & Power & & $\begin{array}{l}\text { Type I } \\
\text { error }\end{array}$ & Power & & $\begin{array}{l}\text { Type I } \\
\text { error }\end{array}$ & Power \\
\hline \multirow[t]{4}{*}{0.005} & 5 & 485 & 0.0664 & 0.6432 & 1569 & 0.1028 & 0.8606 & - & - & - & 1037 & 0.0948 & 0.7992 \\
\hline & 10 & 326 & 0.0566 & 0.6968 & 1057 & 0.0784 & 0.9386 & 515 & 0.0704 & 0.8106 & 464 & 0.0704 & 0.7806 \\
\hline & 20 & 282 & 0.0486 & 0.7258 & 917 & 0.0624 & 0.9770 & 336 & 0.0450 & 0.7934 & 331 & 0.0458 & 0.7850 \\
\hline & 40 & 265 & 0.0466 & 0.7572 & 861 & 0.0512 & 0.9918 & 287 & 0.0424 & 0.7842 & 286 & 0.0474 & 0.7706 \\
\hline \multirow[t]{3}{*}{0.02} & 10 & 629 & 0.0904 & 0.6236 & 2043 & 0.0638 & 0.8196 & - & - & - & $|73|$ & 0.0624 & 0.7968 \\
\hline & 20 & 353 & 0.0660 & 0.6546 & 1147 & 0.0614 & 0.8924 & 1852 & 0.0576 & 0.9432 & 677 & 0.0752 & 0.7976 \\
\hline & 40 & 290 & 0.0556 & 0.7008 & 942 & 0.0582 & 0.9486 & 435 & 0.0558 & 0.8092 & 401 & 0.0514 & 0.7960 \\
\hline \multirow[t]{2}{*}{0.05} & 20 & 743 & 0.0562 & 0.6256 & 2414 & 0.0564 & 0.8140 & - & - & - & 2165 & 0.0480 & 0.7976 \\
\hline & 40 & 361 & 0.0604 & 0.6242 & 1173 & 0.0500 & 0.8598 & - & - & - & 770 & 0.0550 & 0.8048 \\
\hline 0.10 & 40 & 652 & 0.0546 & 0.6006 & 2116 & 0.0542 & 0.8090 & - & - & - & $|88|$ & 0.0500 & 0.8036 \\
\hline
\end{tabular}

Sample size calculations were performed considering type I and type II error rates fixed at 0.05 and 0.20 , respectively.

I In some cases, $80 \%$ power was not reachable

with $\bar{m}_{w_{2}}=\frac{2(1-\rho) T^{2}}{g E S^{2}-6.5 \rho T^{2}}$

$V I F_{w_{3}}=\frac{\left(1+\left(4 \bar{m}_{w_{3}}-1\right) \rho\right)\left(1+\left(0.25 \bar{m}_{w_{3}}-1\right) \rho\right)}{1+\left(\bar{m}_{w_{3}}-1\right) \rho}$

with $\bar{m}_{w_{3}}$ being the positive solution of the following equation:

$\bar{m}_{w_{3}}^{2} \rho\left[g E S^{2}-2 \rho T^{2}\right]+\bar{m}_{w_{3}}(1-\rho)\left[g E S^{2}-8.5 \rho T^{2}\right]-2(1-\rho)^{2} T^{2}=0$

$V I F_{d}=1+\left[3.25 \bar{m}_{d}-1\right] \rho$

with $\bar{m}_{d}=\frac{2(1-\rho) T^{2}}{g E S^{2}-6.5 \rho T^{2}}$

The distribution-based and cluster size weights correction are equivalent [21]. We therefore no longer consider the distribution-based correction and focus on the 3 weighted corrections proposed by Kerry et al [3].

\section{Simulation study}

Monte Carlo simulations were performed to determine to what extent the proposed corrections could lead to adequately powered trials. We thus calculated the sample size needed assuming a Pareto repartition, using each of the adjusted VIFs. For each situation, we then simulated cluster randomized trials with a Pareto imbalance to estimate empirical type I error and power. The same approach as that explained in the preceeding was used.

Results

Results are displayed in Tables 3 and 4 for effect sizes of 0.25 and 0.50 , respectively. For the cluster size weights correction, several situations existed in which the sample size calculations showed that $80 \%$ power could not be reached, thus preventing the generation of associated data sets. If sample size calculations were possible, this correction led to sample sizes barely greater than the sample size obtained with the minimal variance weights correction and empirical type I error and power near the nominal value. This result is consistent for the different values of ES, $\rho$ and $g$ in Tables 3 and 4, except for the combination $0.25 / 0.02 / 20$. Actually, for fixed values of ES, couples of values for $(g, \rho)$ lead to null values of the denominator of $m_{w 2}$. If ES is fixed at 0.25 , the couple $(20,0.0233)$ is one of these. For $\rho$ just under this critical value $(0.020$ in our case), $\mathrm{m}_{\mathrm{w} 2}$ begins to diverge, and when $\rho$ is greater, $\mathrm{m}_{\mathrm{w} 2}$ can no longer be calculated. Equal weights correction led to a much greater sample size than minimum variance weights, particularly when the ICC is small, and the empirical power obtained was therefore much higher than its nominal value: it may even reach $99 \%$ if the nominal value were fixed at $80 \%$. The minimum variance weights correction required the smallest increase in sample size and resulted in the smallest difference between empirical and nominal power. Empirical type I errors were also near the nominal 5\% level, except when both the number of clusters and the ICC are small. 
Table 4: Required sample size and empirical Type I error and power when using corrected variance inflation factors with an a priori hypothesized Pareto imbalance in cluster size - Effect size $=0.50$

\begin{tabular}{|c|c|c|c|c|c|c|c|c|c|c|c|c|c|}
\hline \multirow{3}{*}{$\begin{array}{c}\text { Intraclass } \\
\text { correlation } \\
\text { coefficient }(\rho)\end{array}$} & \multirow{3}{*}{$\begin{array}{l}\text { Number of } \\
\text { clusters in } \\
\text { each arm (g) }\end{array}$} & \multicolumn{3}{|c|}{ No correction } & \multicolumn{3}{|c|}{ Equal weights } & \multicolumn{3}{|c|}{ Cluster size weights 1} & \multicolumn{3}{|c|}{ Minimum variance weights } \\
\hline & & \multirow[t]{2}{*}{$\begin{array}{l}\text { Sample } \\
\text { size }\end{array}$} & \multicolumn{2}{|c|}{$\begin{array}{c}\text { Empirical } \\
\text { probabilities }\end{array}$} & \multirow[t]{2}{*}{$\begin{array}{l}\text { Sample } \\
\text { size }\end{array}$} & \multicolumn{2}{|c|}{$\begin{array}{c}\text { Empirical } \\
\text { probabilities }\end{array}$} & \multirow[t]{2}{*}{$\begin{array}{c}\text { Sample } \\
\text { size }\end{array}$} & \multicolumn{2}{|c|}{$\begin{array}{c}\text { Empirical } \\
\text { probabilities }\end{array}$} & \multirow[t]{2}{*}{$\begin{array}{c}\text { Sample } \\
\text { size }\end{array}$} & \multicolumn{2}{|c|}{$\begin{array}{c}\text { Empirical } \\
\text { probabilities }\end{array}$} \\
\hline & & & $\begin{array}{l}\text { Type I } \\
\text { error }\end{array}$ & Power & & $\begin{array}{l}\text { Type I } \\
\text { error }\end{array}$ & Power & & $\begin{array}{l}\text { Type I } \\
\text { error }\end{array}$ & Power & & $\begin{array}{l}\text { Type I } \\
\text { error }\end{array}$ & Power \\
\hline \multirow[t]{4}{*}{0.005} & 5 & 89 & 0.0256 & 0.6250 & 288 & 0.0536 & 0.9330 & 111 & 0.0270 & 0.7156 & 108 & 0.0324 & 0.6906 \\
\hline & 10 & 73 & 0.0352 & 0.7090 & 236 & 0.0528 & 0.9768 & 79 & 0.0306 & 0.7370 & 79 & 0.0306 & 0.7370 \\
\hline & 20 & 67 & 0.0334 & 0.7322 & 218 & 0.0470 & 0.9912 & 70 & 0.0390 & 0.7524 & 70 & 0.0390 & 0.7524 \\
\hline & 40 & 65 & 0.0382 & 0.7518 & 210 & 0.0394 & 0.9970 & 66 & 0.0400 & 0.7558 & 66 & 0.0400 & 0.7558 \\
\hline \multirow[t]{4}{*}{0.02} & 5 & 119 & 0.0674 & 0.6262 & 387 & 0.1072 & 0.8642 & - & - & - & 256 & 0.0954 & 0.7946 \\
\hline & 10 & 81 & 0.0550 & 0.6910 & 261 & 0.0900 & 0.9346 & 127 & 0.0654 & 0.7990 & 115 & 0.0672 & 0.7856 \\
\hline & 20 & 70 & 0.0460 & 0.7056 & 226 & 0.0684 & 0.9752 & 83 & 0.0492 & 0.7680 & 82 & 0.0482 & 0.7680 \\
\hline & 40 & 66 & 0.0422 & 0.7328 & 212 & 0.0534 & 0.9908 & 71 & 0.0390 & 0.7540 & 71 & 0.0390 & 0.7540 \\
\hline \multirow[t]{4}{*}{0.05} & 5 & 423 & 0.0768 & 0.6808 & 1375 & 0.0578 & 0.8130 & - & - & - & 1311 & 0.0556 & 0.7962 \\
\hline & 10 & 103 & 0.0770 & 0.6342 & 335 & 0.0824 & 0.8600 & - & - & - & 230 & 0.0920 & 0.7952 \\
\hline & 20 & 76 & 0.0528 & 0.6620 & 245 & 0.0652 & 0.9284 & 136 & 0.0706 & 0.8252 & 115 & 0.0628 & 0.7872 \\
\hline & 40 & 67 & 0.0516 & 0.7026 & 217 & 0.0590 & 0.9712 & 83 & 0.0578 & 0.7694 & 81 & 0.0488 & 0.7772 \\
\hline \multirow[t]{3}{*}{0.15} & 10 & 213 & 0.0730 & 0.6394 & 691 & 0.0548 & 0.8042 & - & - & - & 631 & 0.0572 & 0.8002 \\
\hline & 20 & 89 & 0.0674 & 0.6276 & 290 & 0.0644 & 0.8646 & - & - & - & 193 & 0.0638 & 0.7888 \\
\hline & 40 & 70 & 0.0536 & 0.6641 & 225 & 0.0564 & 0.9316 & 122 & 0.0506 & 0.8208 & 104 & 0.0578 & 0.7838 \\
\hline
\end{tabular}

In some cases, $80 \%$ power was not reachable

\section{Robustness of sample size adjustment for unbalanced trials with misspecification of the ICC \\ Method}

We assessed the robustness of the different sample size adjustments for Pareto-like unbalanced trials with misspecification of the ICC. We considered an effect size of 0.25 , a priori postulated ICCs of 0.005 and 0.020 and the combinations of number of clusters and cluster sizes previously used (see sample sizes in Table 3). Then, for each weighting method, (i.e., for each total number of subjects of each arm $\mathrm{N}_{\mathrm{w} 1}, \mathrm{~N}_{\mathrm{w} 2}, \mathrm{~N}_{\mathrm{w} 3}$ ) we plotted the expected power calculated for a pre-specified ICC as a function of the real ICC (which will be a posteriori assessed). This power was calculated by use of the variance inflation factor $\mathrm{VIF}_{\mathrm{w} 3}$ derived from minimum variance weights, because it allows for calculating an expected power that does not differ from the empirical one by more than $3.8 \%$ in the situations explored in Table 3 (data not shown). For reference, we also plotted the expected power (calculated with the usual VIF) as a function of the real ICC in cases of no imbalance in cluster size.

\section{Results}

Results are displayed in Figures 1 and 2 for an effect size of 0.25 and a priori postulated ICC values of 0.005 and 0.020 , respectively. As expected [20], in any situation, the power decreases as the ICC increases, and this result is all the more important when the number of clusters is low. In the planning situations explored, minimum variance weights and cluster size weights curves are very close, except when 20 clusters per intervention arm are randomized and the ICC is a priori fixed at 0.020 , but this latter situation is extreme, as discussed previously. Otherwise, the power associated with equal weights remains greater than that associated with minimum variance weights in any situation. However, this finding probably just reflects that the use of this weighting system leads to higher required sample sizes than the use of a minimum variance weights system (cf Tables 3 and 4) and therefore higher power. In any case, imbalance in cluster size is associated with a higher sensitivity to the a priori-specified ICC than constant cluster size. For example, let us consider the case of 20 clusters per intervention arm: if the ICC is a priori postulated at 0.005 , but in reality equals 0.015 , the power associated with constant cluster size decreases from 0.80 to 0.75 only, whereas the power associated with Pareto repartition decreases from 0.80 to 0.68 (with the minimum variance weighting system). However, all weighting systems show great sensitivity to the actual value of the ICC. Consider the former example ( $\mathrm{ES}=0.25, \mathrm{~g}=20$ and Pareto repartition, increase in ICC from 0.005 to 0.015 ), the power associated with equal weights will decrease from 0.98 to 0.90 , and the power associated with cluster size weights from 0.80 to 0.68 . Thus, if little prior knowledge is available concerning the value of the ICC, the sensitivity analysis involving several values of ICC is of major importance, particularly when imbalance in cluster size is expected. 

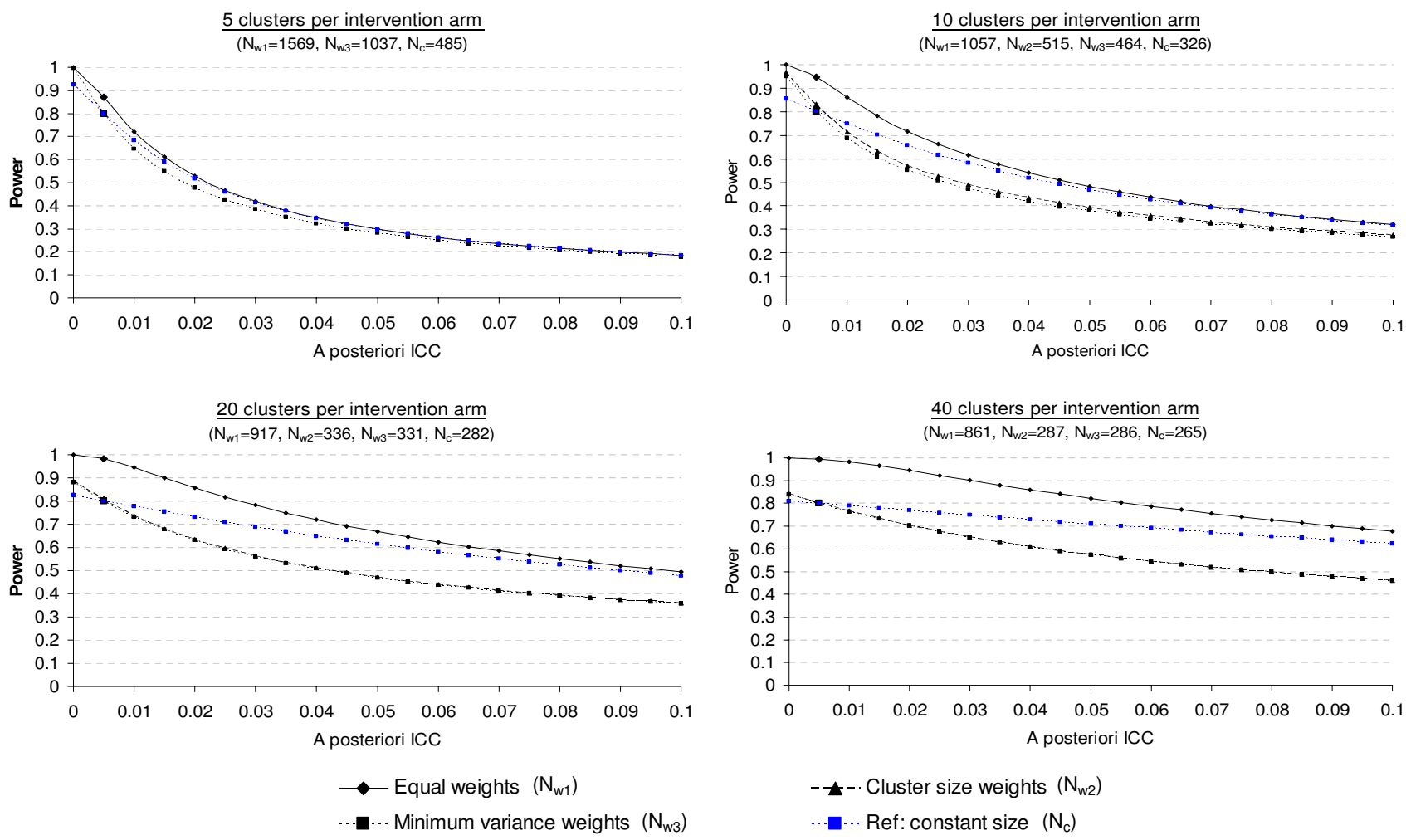

Figure I

Real power of cluster randomized trials according to the discrepancy between the a priori postulated and a posteriori estimated intraclass correlation coefficients (ICCs). The ICC is a priori postulated at 0.005 and sample sizes (N) and associated powers were calculated: $\mathrm{I}^{\circ}$ ) assuming Pareto repartition of cluster sizes and using 3 corrections of the variance inflation factor (equal weights, cluster size weights and minimum variance weights), $2^{\circ}$ ) assuming constant cluster size (reference).

\section{Practical implications}

General considerations

Cluster size inequality may induce a loss of power and must be taken into account at the planning stage by using the minimum variance weights correction. From a practical point of view, 2 situations must be distinguished. First, when entire clusters are randomized such as in clustercluster trials [22]. the cluster size distribution is a priori known and cluster size inequalities are therefore easy to be taken into account at the planning stage. Second, if physicians have to recruit patients to each cluster according to selection criteria, cluster size distribution cannot $a$ priori be known. In this latter situation, a sensitivity analysis must be performed considering several hypotheses on cluster size distribution for an optimal sample size determination.

\section{Adaptation of the VIF for a Pareto like imbalance}

Let us assume that the cluster size inequality corresponds to a Pareto-like distribution, say that in each arm a proportion $(\gamma)$ of clusters actually recruit the proportion $(\tau)$ of patients to be recruited (which implies $\gamma \leq \tau$ ). If $\gamma$ and $\tau$ are fixed at $20 \%$ and $80 \%$, respectively, we have the Pareto imbalance defined previously; if $\gamma$ and $\tau$ are equal, the cluster size imbalance is absent or moderate (and can then be neglected). The sensitivity analysis then consists of varying the parameters $(\gamma)$ and $(\tau)$, thus allowing for imbalance increases with the absolute difference between the 2 values. The inflation factor calculated with the minimum variance weights correction will be the following (Appendix B):

$$
V I F=\frac{\left[1+\left(\frac{1-\tau}{1-\gamma} m-1\right) \rho\right]\left[1+\left(\frac{\tau}{\gamma} m-1\right) \rho\right]}{\tau\left[1+\left(\frac{1-\tau}{1-\gamma} m-1\right) \rho\right]+(1-\tau)\left[1+\left(\frac{\tau}{\gamma} m-1\right) \rho\right]}
$$

To illustrate the discrepancy between nominal and real power if an imbalance of the form " $\gamma$ clusters actually recruit $\tau$ patients" is not taken into account, we performed the following calculations. We used formula (4) (i.e., assuming a constant cluster size) to derive the number of subjects needed. Then, using expression (9), we calculated the expected power with such a sample size, with a pro- 

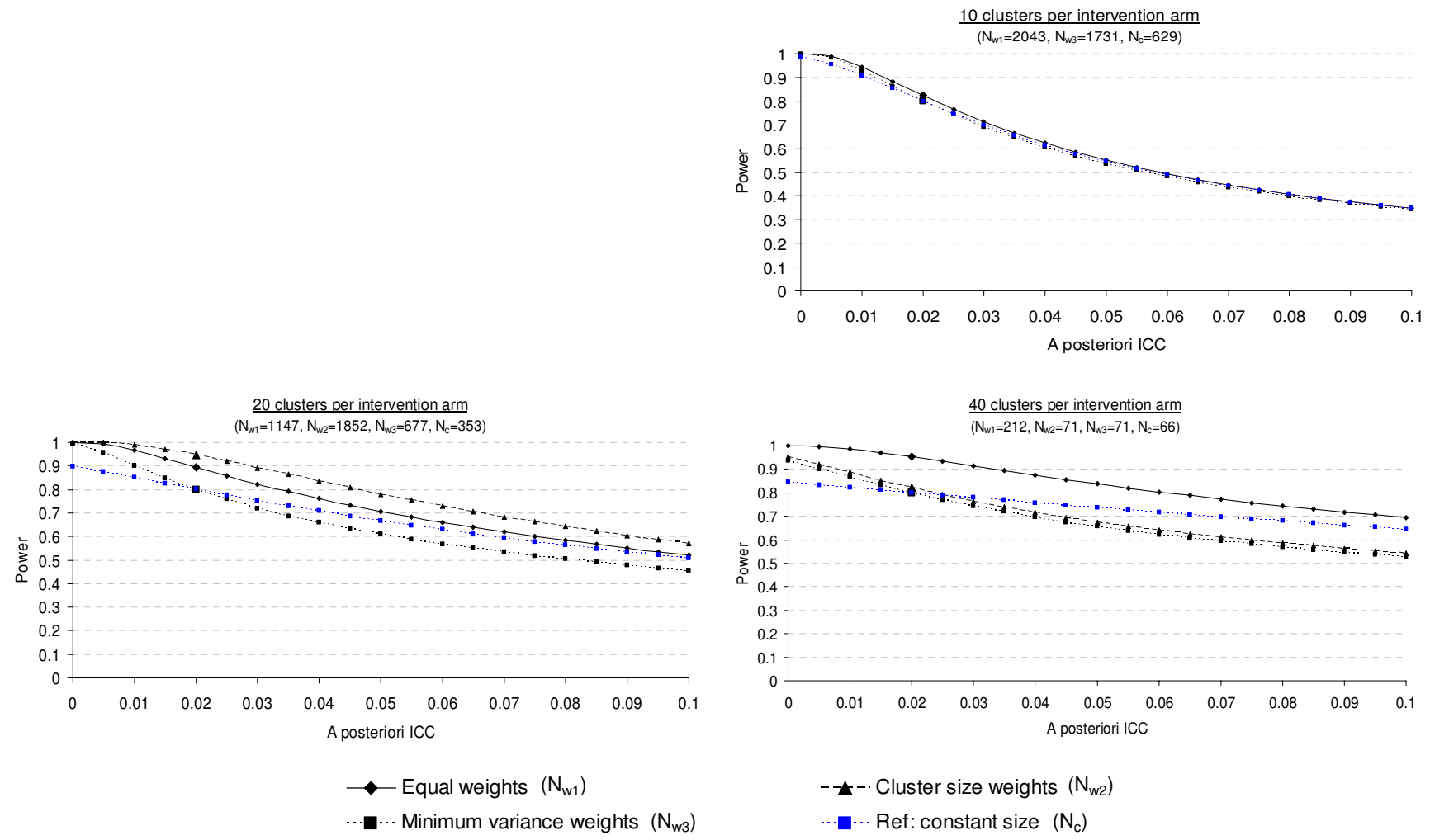

\section{Figure 2}

Real power of cluster randomized trials according to the discrepancy between the a priori postulated and a posteriori estimated intraclass correlation coefficients (ICCs). The ICC is a priori postulated at 0.020 and sample sizes (N) and associated powers were calculated: $\mathrm{I}^{\circ}$ ) assuming Pareto repartition of cluster sizes and using 3 corrections of the variance inflation factor (equal weights, cluster size weights and minimum variance weights), $2^{\circ}$ ) assuming constant cluster size (reference).

portion of $\gamma$ clusters actually recruiting a proportion $\tau$ of the patients to be included.

Figures 3 and 4 display the results for several combinations of ES/ICC/g and $\gamma / \tau$ under the assumption of no empty cluster. The upper part of Figures 3 and 4 is empty, since an $80 \%$ power cannot be reach for the associated combinations of ICC and g. Moreover, $\gamma$ is smaller than or equal to $\tau$, which explains why any upper part of matrices associated with an ICC/g combination is empty. As expected, the bigger the cluster size inequality, the more important the discrepancy between nominal and real power. For example, let us consider a trial aimed at detecting a 0.25 effect size in which 10 clusters are to be randomized in each arm. Assuming an ICC of 0.005 and a balance in cluster size, this study would require 326 subjects to be recruited in each arm to reach $80 \%$ power. If $10 \%$ of the clusters recruit $50 \%$ of the subjects, the power barely declines, to $77 \%$; if a major imbalance such as $90 \%$ of the patients are to be recruited by $10 \%$ of the clusters, the power would fall to $54 \%$. The latter phenomenon is all the more acute with a low number of clusters; critical situations in which a substantial loss in power may be expected are displayed in Figures 3 and 4. Red levels approximately follow diagonals representing constant $\tau$ - $\gamma$ differences. It can be shown (appendix $\mathrm{C}$ ) that the gini coefficient, a quantitative measure of site accrual inequality [23], comes down to the absolute difference between $\tau$ and $\gamma$ when a proportion $\gamma$ of clusters actually recruit a proportion $\tau$ of patients to be recruited. Our results show that varying this summary measure of imbalance is enough for performing a sensitivity analysis and that there is no need to specify both $\tau$ and $\gamma$.

Assigning a value of 1 to $\tau$ creates a situation in which a proportion $(1-\gamma)$ of clusters is empty. In this situation achieving the required sample size supposes to increase the average cluster size of the $\gamma g$ clusters by a factor $1 / \gamma$. However one has to be aware that such a strategy will indeed allow achieving the pre-specified sample size, but it will not allow to reach the nominal power. Indeed it is known that for a fixed total number of subjects, the higher the number of clusters, the higher the power [1] which means that reducing the number of clusters will translate 


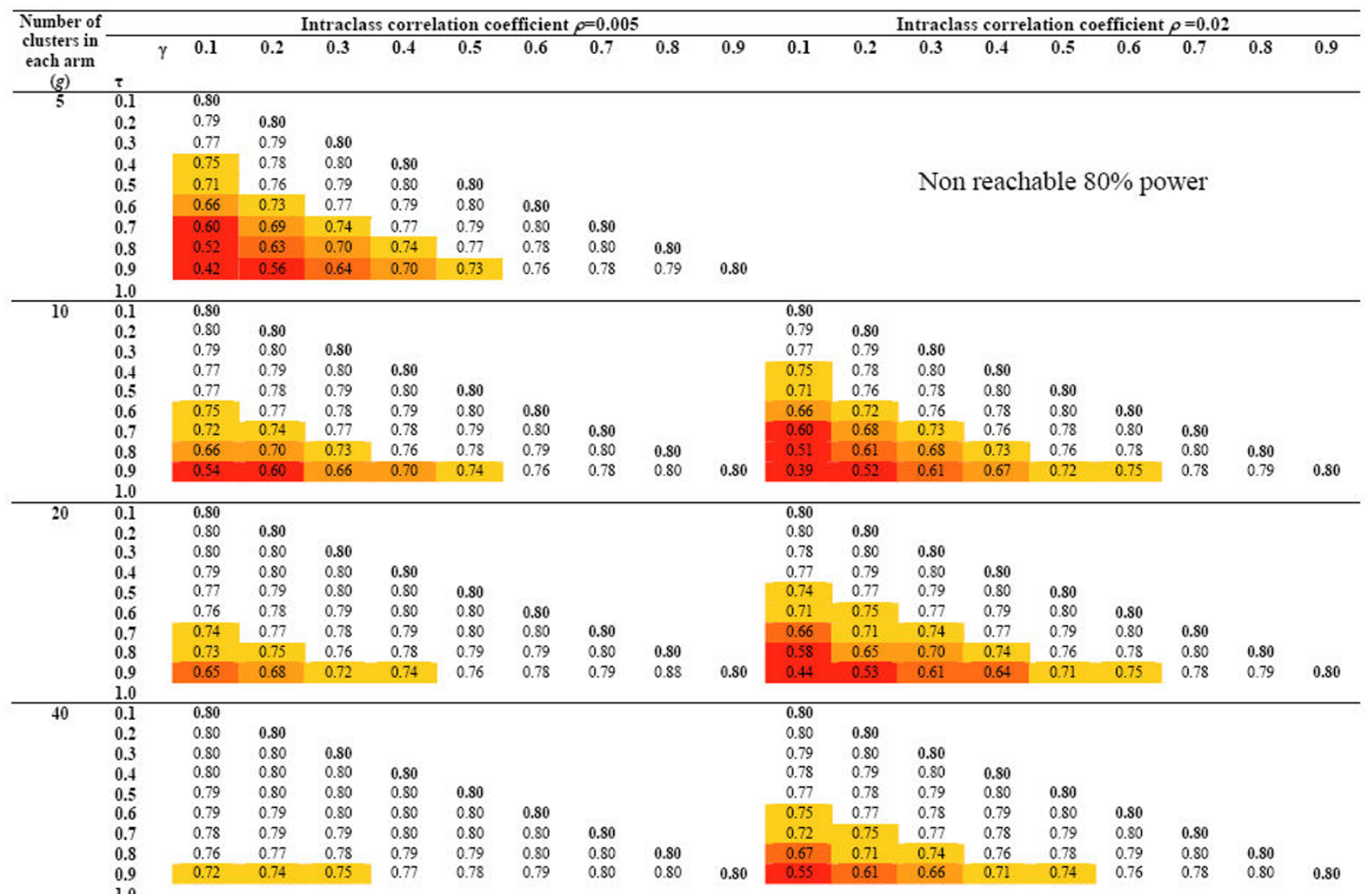

$\gamma$ is the proportion of big clusters and $\tau$ the proportion of patients in big clusters

The effect size to be detected was fixed at 0.25 , the nominal values for type I and type II error rates were fixed at 0.05 and 0.20 , respectively.

$$
\begin{array}{lll}
1-\beta>0.75 & \square, 75 \geq 1-\beta>0.70 \quad \square 0.70 \geq 1-\beta>0.65 \quad \square 0.65 \geq 1-\beta>0.60 \quad \square 0.60 \geq 1-\beta
\end{array}
$$

\section{Figure 3}

Power of cluster randomized trials if an imbalance in cluster size is not taken into account when planning. The imbalance is $a$ priori hypothesized to be "a proportion of $\gamma$ clusters will actually recruit a proportion $\tau$ of the subjects to be included" $(\gamma \leq \tau)-$ The intraclass correlation coefficient is fixed at 0.005 and 0.02 .

in a loss in power even if the pre-specified sample size is achieved. Therefore, in case it is anticipated that empty clusters may occur, sensitivity analyses have to be conducted using formula (4) on the basis of the hypothesized number of active clusters $\mathrm{g}^{\prime}=\gamma \mathrm{g}$.

\section{Discussion}

A moderate inequality in cluster sizes has little effect on power and can thus be neglected at the planning stage. However, a major imbalance in cluster sizes, like the "Pareto" imbalance, (i.e. $80 \%$ of the subjects belong to only $20 \%$ of the clusters) is associated with a loss in power, and the phenomenon is all the more important when the number of clusters is low and/or the ICC is high. In these situations, the minimum variance weights correction has good properties and allows for achieving the nominal power. This result, obtained in the extreme situation of a Pareto imbalance, suggests that this correction can be used to derive sample size or power in any situation where, in each group, cluster sizes can be separated in two strata, the small cluster stratum and the big cluster stratum. The higher sensitivity of severely unbalanced trials to the a priori-postulated value of the ICC compared to that of balanced trials emphasized the necessity of a sensitivity analysis on this parameter. We derived an adaptation of the VIF, which should be used when the imbalance is a priori hypothesized to be "a proportion of $\gamma$ clusters will actually recruit a proportion $\tau$ of the subjects to be included".

A limit to this approach remains the degree of imbalance being usually difficult to foresee at the planning stage, 


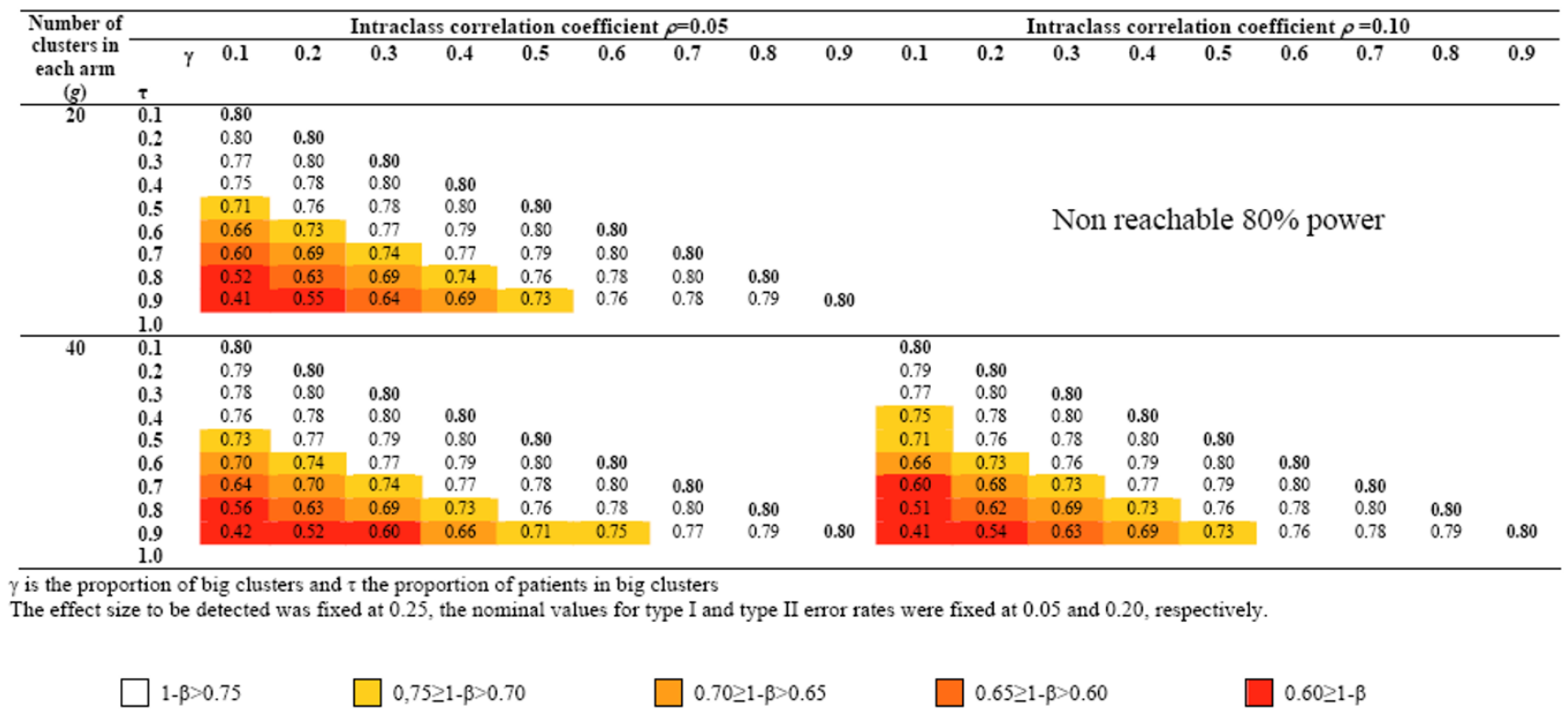

\section{Figure 4}

Power of cluster randomized trials if an imbalance in cluster size is not taken into account when planning. The imbalance is $a$ priori hypothesized to be "a proportion of $\gamma$ clusters will actually recruit a proportion $\tau$ of the subjects to be included" $(\gamma \leq \tau)-$ The intraclass correlation coefficient is fixed at 0.05 and 0.10 .

except when, for instance, families or practices are randomized and clusters as a whole are included in the trial. In these latter situations, one may a priori know precisely the cluster size repartition and therefore use the minimum variance weights correction as initially specified by Kerry et al [3]. However, if, within each cluster, the physician has to recruit patients to be included in the trial, cluster size distribution may then be difficult to hypothesize. It is all the more difficult since cluster sizes are usually not reported in published clustered randomized trials. We therefore proposed to consider that cluster sizes distribution can be divided in each arm in two strata: a stratum of small clusters, and another of large clusters. This hypothesis may be debatable. However, since a moderate inequality of cluster size is of minor effect, it seems a rather useful and simple way to consider the risk of cluster size inequality at the planning stage, particularly since no pre-

Table 5:

\begin{tabular}{lccc}
\hline & $\begin{array}{c}\text { Number of } \\
\text { clusters by } \\
\text { intervention arm }\end{array}$ & $\begin{array}{c}\text { Number of } \\
\text { patients } \\
\text { belonging to } \\
\text { the clusters }\end{array}$ & Mean cluster size \\
\hline $\begin{array}{l}\text { Small } \\
\text { clusters }\end{array}$ & $0.8 g$ & $0.2 \bar{m} g$ & $(0.2 \bar{m} g) /(0.8 g)=0.25 \bar{m}$ \\
$\begin{array}{l}\text { Big } \\
\text { clusters }\end{array}$ & $0.2 g$ & $0.8 \bar{m} g$ & $(0.8 \bar{m} g) /(0.2 g)=4 \bar{m}$ \\
\hline
\end{tabular}

cise data on cluster size inequality are available. Another limitation is that our work focused on normally distributed continuous outcomes. More work is needed to extend our results to non-normal distributions, especially with binary variables. Finally, we restricted our work to cases of no differential recruitment between arms, thus considering that imbalance is the same in the two arms. Such a hypothesis may be questionable in cluster randomized trials: since inclusion is posterior to randomization, this may indeed induce differential recruitment and imbalance in patient characteristics, which may lead to questioning the results of the study [24].

Table 6:

\begin{tabular}{lccc}
\hline & $\begin{array}{c}\text { Number of } \\
\text { clusters by } \\
\text { intervention arm }\end{array}$ & $\begin{array}{c}\text { Number of patients } \\
\text { belonging to the } \\
\text { clusters }\end{array}$ & Mean cluster size \\
\hline $\begin{array}{l}\text { Small } \\
\text { clusters }\end{array}$ & $(1-\gamma) g$ & $(1-\tau) \bar{m} g$ & $\frac{1-\tau}{1-\gamma} \bar{m}$ \\
$\begin{array}{l}\text { Big } \\
\text { clusters }\end{array}$ & $\gamma g$ & $\tau \bar{m} g$ & $\frac{\tau}{\gamma} \bar{m}$
\end{tabular}




\section{Conclusion}

In conclusion, our study demonstrates that severely imbalanced trials with continuous outcomes may be highly underpowered. If such imbalance in cluster size can be anticipated at the design stage, minimum variance weights correction should be used to inflate the required sample size. A priori estimation of the expectable imbalance would be facilitated if more details on cluster sizes were given in published cluster randomized trials, as was recently advised in the extension of the CONSORT statement for cluster randomized trials [25]. Moreover, such publication of cluster sizes would be of particular interest to assess the real power of the trial conducted.

\section{Competing interests}

The author(s) declare that they have no competing interests.

\section{Authors' contributions}

This study was designed by LG, BG and PR. LG performed the statistical analysis and drafted the article, which was then revised by BG and PR.

\section{Appendix A: corrected variance inflation factor} (VIF) for an a priori postulated Pareto imbalance Four corrections have been proposed for adjusting sample size in cases of imbalance in cluster size. Considering the specific situation of a Pareto imbalance, the general form of these corrections can be simplified.

\section{Characteristics of the Pareto imbalance}

$g$ refers to the number of clusters within each arm and $\bar{m}$ is the average cluster size

Equal weights (denoted $w_{1}$ ) [3]

With an equal weights correction, the VIF is expressed as:

$V I F_{w_{1}}=\frac{\bar{m}}{g} \sum_{j=1}^{g} \frac{1}{m_{j}}(1-\rho)+\bar{m} \rho$ where $\bar{m}=\frac{1}{g} \sum_{j=1}^{g} m_{j}$

With a Pareto imbalance, this equation is expressed as:

$$
\begin{aligned}
V I F_{w_{1}} & =\frac{\bar{m}_{w_{1}}}{g}\left(\frac{0.8 g}{0.25 \bar{m}_{w_{1}}}+\frac{0.2 g}{4 \bar{m}_{w_{1}}}\right)(1-\rho)+\bar{m}_{w_{1}} \rho \\
& =3.25+\left(\bar{m}_{w_{1}}-3.25\right) \rho
\end{aligned}
$$

where $\bar{m}_{w_{1}}$ (the average cluster size for which an equal weights correction is used) is defined as: $\bar{m}_{w_{1}} g=\frac{2 T^{2}}{E S^{2}}\left[3.25+\left(\bar{m}_{w_{1}}-3.25\right) \rho\right]$

which leads to :

$\bar{m}_{w_{1}}=\frac{6.5(1-\rho) T^{2}}{g E S^{2}-2 \rho T^{2}}$,

where $E S$ refers to the effect size and $T=t_{(1-\alpha / 2), 2(g-1)}+t_{(1}$ $-\beta), 2(g-1)$

Cluster size weights (denoted $w_{2}$ ) [3]

$V I F_{w_{2}}=1+\left(\overline{m_{A}}-1\right) \rho$ where $\overline{m_{A}}=\frac{\sum_{j=1}^{g} m_{j}^{2}}{\sum_{j=1}^{g} m_{j}}$

With a Pareto imbalance, we can write the equation as:

$\bar{m}_{A}=\frac{0.8 g\left(0.25 \bar{m}_{w_{2}}\right)^{2}+0.2 g\left(4 \bar{m}_{w_{2}}\right)^{2}}{\bar{m}_{w_{2}} g}=3.25 \bar{m}_{w_{2}}$

So the VIF is reduced to:

$V I F_{w_{2}}=1+\left(3.25 \bar{m}_{w_{2}}-1\right) \rho$

and

$\bar{m}_{w_{2}}=\frac{2(1-\rho) T^{2}}{g E S^{2}-6.5 \rho T^{2}}$

Minimum variance weights (denoted $w_{3}$ ) [3]

$V I F_{w_{3}}=\frac{\bar{m} g}{\sum_{j=1}^{g} \frac{m_{j}}{1+\left(m_{j}-1\right) \rho}}$

With a Pareto imbalance, the equation can be written as:

$$
\begin{aligned}
V I F_{w_{3}} & =\frac{\bar{m}_{w_{3}} g}{0.8 g \frac{0.25 \bar{m}_{w_{3}}}{1+\left(0.25 \bar{m}_{w_{3}}-1\right) \rho}+0.2 g \frac{4 \bar{m}_{w_{3}}}{1+\left(4 \bar{m}_{w_{3}}-1\right) \rho}} \\
& =\frac{\left(1+\left(4 \bar{m}_{w_{3}}-1\right) \rho\right)\left(1+\left(0.25 \bar{m}_{w_{3}}-1\right) \rho\right)}{1+\left(\bar{m}_{w_{3}}-1\right) \rho}
\end{aligned}
$$


with

$\bar{m}_{w_{3}} g=\frac{2 T^{2}}{E S^{2}}\left[\frac{\left(1+\left(4 \bar{m}_{w_{3}}-1\right) \rho\right)\left(1+\left(0.25 \bar{m}_{w_{3}}-1\right) \rho\right)}{1+\left(\bar{m}_{w_{3}}-1\right) \rho}\right]$

which leads to $\bar{m}_{w_{3}}$ being the positive solution of the following equation:

$\bar{m}_{w_{3}}^{2} \rho\left[g E S^{2}-2 \rho T^{2}\right]+\bar{m}_{w_{3}}(1-\rho)\left[g E S^{2}-8.5 \rho T^{2}\right]-2(1-\rho)^{2} T^{2}=0$

Distribution-based correction (denoted d) [4]

$$
\begin{aligned}
V I F_{d} & =1+\left(\frac{E(m)^{2}+\operatorname{var}(m)}{E(m)}-1\right) \rho \\
\operatorname{var}(m) & =\frac{0.8 g\left(0.25 \bar{m}_{d}\right)^{2}+0.2 g\left(4 \bar{m}_{d}\right)^{2}-\frac{\left(0.8 g\left(0.25 \bar{m}_{d}\right)+0.2 g\left(4 \bar{m}_{d}\right)\right)^{2}}{g}}{g} \\
& =2.25 \bar{m}_{d}^{2}
\end{aligned}
$$

So we have:

$$
\begin{aligned}
V I F_{d} & =1+\left[\frac{\bar{m}_{d}^{2}+2.25 \bar{m}_{d}^{2}}{\bar{m}_{d}}-1\right] \rho \\
& =1+\left[3.25 \bar{m}_{d}-1\right] \rho
\end{aligned}
$$

with

$\bar{m}_{d} g=\frac{2 T^{2}}{E S^{2}}\left[1+\left(3.25 \bar{m}_{d}-1\right) \rho\right]$

that is to say:

$$
\bar{m}_{d}=\frac{2(1-\rho) T^{2}}{g E S^{2}-6.5 \rho T^{2}}
$$

One then recognizes the results obtained using the cluster size weights correction.

\section{Appendix B: minimum variance weights- corrected variance inflation factor (VIF) for an a priori postulated Pareto-like imbalance}

Characteristics of the Pareto-like imbalance

$g$ refers to the number of clusters within each arm and $\bar{m}$ is the average cluster size

Minimum variance weighs VIF

$$
\begin{aligned}
V I F_{w_{3}}= & \frac{\bar{m} g}{\sum_{j=1}^{g} \frac{m_{j}}{1+\left(m_{j}-1\right) \rho}} \\
= & \frac{\bar{m} g}{(1-\gamma) g \frac{\frac{1-\tau}{1-\gamma} \bar{m}}{1+\left(\frac{1-\tau}{1-\gamma} \bar{m}-1\right) \rho}+\gamma g \frac{\frac{\tau}{\gamma} \bar{m}}{1+\left(\frac{\tau}{\gamma} \bar{m}-1\right) \rho}}
\end{aligned}
$$

So we obtain:

$V I F=\frac{\left[1+\left(\frac{1-\tau}{1-\gamma} m-1\right) \rho\right]\left[1+\left(\frac{\tau}{\gamma} m-1\right) \rho\right]}{\tau\left[1+\left(\frac{1-\tau}{1-\gamma} m-1\right) \rho\right]+(1-\tau)\left[1+\left(\frac{\tau}{\gamma} m-1\right) \rho\right]}$

\section{Appendix C: gini coefficient for an a priori} postulated Pareto-like imbalance

$$
\text { gini }=\frac{1}{2 g^{2} \bar{m}} \sum_{i=1}^{g} \sum_{j=1}^{g}\left|m_{i}-m_{j}\right|
$$

Given the characteristics of the Pareto-like imbalance presented in appendix B, considering that clusters are ordered hierarchically by increasing size, the matrix of the difference $\left|m_{i}-m_{j}\right|$ can be written as:

$$
M=\left(\begin{array}{cc}
0_{(\gamma g, \gamma g)} & 1_{(\gamma g,(1-\gamma) g)}\left|\frac{\tau-\gamma}{\gamma(1-\gamma)}\right| \\
1_{((1-\gamma) g, \gamma g)}\left|\frac{\tau-\gamma}{\gamma(1-\gamma)}\right| & 0_{((1-\gamma) g,(1-\gamma) g)}
\end{array}\right)
$$

Where $0_{(\gamma g, \gamma g)}$ and $0_{((1-\gamma) g,(1-\gamma) g)}$ are squared matrices of size $\gamma g$ and $(1-\gamma) g$ respectively and $1_{(\gamma g,(1-\gamma) g)}$ and $1_{((1-\gamma) g, \gamma g)}$ are matices of size $\gamma g \times(1-\gamma) g$ and $(1-\gamma) g \times \gamma g$ respectively, containing only $1 \mathrm{~s}$.

Thus:

$$
\begin{aligned}
& \text { gini }=\frac{1}{2 g^{2} \bar{m}} 2 g^{2} \gamma(1-\gamma)\left|\frac{\tau-\gamma}{\gamma(1-\gamma)}\right| \bar{m} \\
& \text { gini }=|\tau-\gamma|
\end{aligned}
$$

\section{Acknowledgements}

The work was funded by a grant from the Foundation for Medical research (FRM). 


\section{References}

I. Murray DM: Design and Analysis of Group-Randomized Trials Oxford University Press: New York; 1998.

2. Donner A, Klar N: Design and Analysis of Cluster Randomization Trials in Health Research Arnold: London; 2000.

3. Kerry SM, Bland JM: Unequal cluster sizes for trials in English and Welsh general practice: implications for sample size calculations. Stat Med 200I, 20:377-390.

4. Manatunga AK, Hudgens MG, Chen S: Sample size estimation in cluster randomized studies with varying cluster size. Biometrical Journal 200I, I:75-86.

5. Hannan PJ, Murray DM, Jacobs DR, McGovern PG: Parameters to aid in the design and analysis of community trials: intraclass correlations from the Minnesota Heart Health Program. Epidemiology 1994, 5:88-95.

6. Martinson BC, Murray DM, Jeffery RW, Hennrikus DJ: Intraclass correlation for measures from a worksite health promotion study: estimates, correlates, and applications. Am J Health Promot 1999, I 3:347-357.

7. Murray DM, Short BJ: Intraclass correlation among measures related to tobacco use by adolescents: estimates, correlates, and applications in intervention studies. Addict Behav 1997 22:I-12.

8. Murray DM, Clark MH, Alexander CW: Intraclass correlation from a community-based alcohol prevention study: the effect of repeat observations on the same communities. J Stud Alcohol 2000, 61:881-90.

9. Murray DM, Phillips GA, Bimbaum AS, Lytle LA: Intraclass correlation for measures from a middle school nutrition intervention study: estimates, correlates, and applications. Health Educ Behav 200I, 28:666-679.

10. Siddiqui O, Hedeker D, Flay BR, Hu FB: Intraclass correlation estimates in a school-based smoking prevention study. Am J Epidemiol 1996, I44:425-433.

II. Smeeth L, Siu-Woon NgE: Intraclass correlation coefficients for cluster randomized trials in primary care: data from the MRC Trial of the Assessment and Management of Older People in the Community. Control Clin Trials 2002, 23:409-42I.

12. Reading R, Harvey I, McLean M: Cluster randomised trials in maternal and child health: implications for power and sample size. Arch Dis Child 2000, 82:79-83.

13. Eldridge SM, Ashby D, Feder GS, Rudnicka AR, Ukoumunne OC: Lessons for cluster randomized trials in the twenty-first century a systematic review of trials in primary care. Clinical Trials 2004, I:80-90.

14. Ukoumunne OC, Gulliford MC, Chinn C, Sterne JAC, Burney PG] Methods for evaluating area-wide and organisation-based interventions in health and health care: a systematic review. Health Technol Assess 1999, 3:iii-92.

15. Gulliford MC, Adams G, Ukoumunne OC, Latinovic R, Chinn S Campbell MJ: Intraclass correlation coefficient and outcome prevalence are associated in clustered binary data. I Clin Epidemiol 2005, 58:246-25I.

16. Ukoumunne OC: A comparison of confidence interval methods for the intraclass correlation coefficient in cluster randomized trials. Stat Med 2002, 2 I:3757-3774.

17. Bellamy SL, Gibberd R, Hancock L, Howley P, Kennedy B, Klar N, Lipsitz S: Analysis of dichotomous outcome data for community intervention studies. Stat Methods Med Res 2000, 9:135-I59.

18. Verbeke G, Molenberghs G: Linear Mixed Models in Practice. A SAS Oriented Approach Springer-Verlag: New York; 1997.

19. Littell RC, Milliken GA, Stroup WW, Wolfinger RD: SAS System for Mixed Models BBU Press: Cary; 1996.

20. Guittet L, Giraudeau B, Ravaud P: A priori postulated and real power in cluster randomized trials: mind the gap. $B M C M e d$ Res Methodol 2005, 5:25

21. Lake S, Kammann E, Klar N, Betensky R: Sample size re-estimation in cluster randomization trials. Stat Med 2002, 21:1337-1350.

22. Edwards SJ, Braunholtz DA, Lilford RJ, Stevens AJ: Ethical issues in the design and conduct of cluster randomised controlled trials. $B M J$ | 999, 3 I 8: |407-| 409.

23. Haidich $A B$, loannidis JPA: The Gini coefficient as a measure for understanding accrual inequalities in multicenter clinical trials. J Clin Epidemiol 2004, 57:34 I-8.
24. Puffer S, Torgerson D, Watson J: Evidence for risk of bias in cluster randomised trials: review of recent trials published in three medical journals. BMJ 2003, 327:785-789.

25. Campbell MK, Elbourne DR, Altman DG: CONSORT statement: extension to cluster randomised trials. BMJ 2004, 328:702-708.

\section{Pre-publication history}

The pre-publication history for this paper can be accessed here:

http://www.biomedcentral.com/1471-2288/6/17/prepub
Publish with Bio Med Central and every scientist can read your work free of charge

"BioMed Central will be the most significant development for disseminating the results of biomedical research in our lifetime. "

Sir Paul Nurse, Cancer Research UK

Your research papers will be:

- available free of charge to the entire biomedical community

- peer reviewed and published immediately upon acceptance

- cited in PubMed and archived on PubMed Central

- yours - you keep the copyright
BioMedcentral 